\title{
Japanese Salient Perceptions of Ubiquitous Monitoring
}

\author{
Stuart Moran \\ Mixed Reality Lab, \\ University of Nottingham, \\ Nottingham, UK \\ stuart.moran@nottingham.ac.uk
}

\author{
Toyoaki Nishida \\ Nishida Lab, \\ Kyoto University, \\ Kyoto, Japan \\ nishida@i.kyoto-u.ac.jp
}

\author{
Keiichi Nakata \\ Informatics Research Centre, \\ University of Reading, \\ Reading, UK \\ k.nakata@henley.reading.ac.uk
}

\begin{abstract}
Ubiquitous Computing systems stand to bring many benefits in life. To achieve this, such systems will require unprecedented levels of data to be collected, in real time, about users. One issue with this level of data collection is that monitoring can often cause users to change their behavior, potentially rendering the data collected inaccurate. The PSA-BI model was developed to predict the behavior/responses of users to a monitoring system based on the causal relationships between system characteristics, perceptions and behavioral intention. This paper presents a study exploring how Japanese office workers in Kyoto University perceive and react to a wearable Ubiquitous Monitoring device.
\end{abstract}

Keywords - Attitudes;Japan;Modelling; Monitoring;Prediction;

\section{INTRODUCTION}

A world of Ubiquitous Computing (UC), full of networked mobile and embedded technologies is approaching. The benefits of this technology are numerous, and act as the major driving force behind its development. These benefits are brought about, in part, by Ubiquitous Monitoring (UM): the continuous and wide spread collection of significant amounts of data about users [1]. While UM is a source of many of the benefits of the technology, it may also play a role in its failure. To appreciate the potential negative aspects of this new type of monitoring, we must look at existing monitoring technologies and methods such as: closed circuit television cameras, human observation, health-based systems in hospitals and automated city travel passes. These systems share a common social implication where they can often be found to undesirably influence the behavior of those using them (i.e. being observed) [2-5]; in addition to causing feelings of stress and distrust [2].

In light of this, we anticipate that both behavioral change and other undesirable effects are likely to continue to occur in UM systems. Furthermore, given the reduction in physical constraints (e.g. walls, floors and distance) that hinder existing technologies, the effects of the monitoring are likely to become amplified. The problem this creates for the field of UC is that if the system used to collect naturalistic data about users is influencing their behaviors and cognitive state, then the data collected is unlikely to be accurate. As a result, the system may provide a sub-optimal response or, at worst, suffer a failure.

In response to this issue, a number of predictive models have been developed with the aim of minimizing or preventing undesirable behaviors prior to a UM systems development and implementation. Such models would allow designers to explore the potential behavioral impacts of their system designs without costly developments and negative social reactions; which could resolve many social issues before they occur. One of the implications of such tools is the ability for designers to potentially manipulate the behaviors of users of their systems. This arguably leaves the responsibility and assessment of the morality surrounding the outcomes of the monitoring system in the hands of the designer.

\section{EXISTING FRAMEWORKS}

The ubiquitous computing acceptance model [6], and the awareness monitoring model [7], are highly relevant existing frameworks proposed to help guide feature research or model the undesirable effects of pervasive computer systems. While both are useful for conceptualizing the problem space, they fail to consider many of the core aspects of UM and behavioral change. The Perceptions of System Attributes Behavioral Intention (PSA-BI) model was developed to address these issues [1].

Like the above models, the PSA-BI model is grounded in aspects of sociological theories such as the Theory of Planned Behavior (TPB) [8] and Technology Acceptance Model [9]. What differentiates this model is the direct focus on UM technology, and the clear identification of specific behavioral influencing system characteristics. The complete model, which can be found in [1], details how system characteristics (e.g. device intrusiveness, data types, degrees of user control) relate to, and can influence, user perceptions of a monitoring system. These perceptions are then used as a bridge to the TPB, acting as antecedents of attitudes, which in turn precede behavioral intentions and behavior [8]. In short, the model directly relates objective and subjective factors to user behaviors and responses.

While the model has been applied in a number of domains such as UC [1], assistive technologies [10] and persuasion

This work was supported by a research grant from the Japanese Society for the Promotion of Science (JSPS), Japan. 
[11], the impact of culture as an exogenous moderating variable has yet to be explored. One of the motivations for exploring this variable in particular (as opposed to age or gender) is an anticipated strong difference between people from different countries and cultures in their views of monitoring systems.

\section{STUDY DESIGN}

Japan as a country has a unique and intriguing culture, and as such is an ideal environment to explore the potential impact of culture on user perceptions, particularly as the PSA-BI model was based on data collected primarily in the UK. One of the notable aspects of Japanese culture is the general positive attitude towards technology adoption e.g. iMode [12]. Given that monitoring or surveillance in the Japanese public sphere is less pervasive than in other countries such as the UK [13] (likely due to low crime rates in Japan [14]) their experience of large scale monitoring systems is likely to be limited. Further to this, it is interesting to note that some studies have suggested there is no direct translation for the word 'privacy' in Japanese [15]. This might partly reflect the state of Japanese attitudes and perceptions towards data collection and use; although does not necessarily mean the concept of privacy does not exist in modern Japan. With regards to Japanese workplaces and monitoring, they are commonly large open plan offices (e.g. [16]) and as such there exists a 'direct' form of observation; though it may not necessarily be perceived this way.

To explore the influence of culture on the components of the PSA-BI model, a two-part study has been designed. The first part of the study was carried out in Kyoto University, Japan, and is reported on in this paper. This is to be followed by an analysis and comparison of a replicated study in the University of Reading, UK.

When studying user responses and behaviors involving a technology, their perceptions of the technology are of particular importance to understanding their actions. This is because these user's actions are grounded in their perceptions of reality, and not the reality itself. Hence perceptions of a system's characteristics are arguably more important than the characteristics themselves, particularly salient perceptions. These are the immediate perceptions (or attitudes) that a user has about a technology based purely on their previous experiences, with no other information available. For example, when you hear a song for the first time, your strongest initial responses to it can be described as salient. In terms of technology acceptance, perceptions such as ease of use and usefulness are frequently described as salient [17] [18]. Salient perceptions are of critical importance because they will likely influence the users subsequent experiences with the technology, and thus play a significant part in determining system acceptance and use [19].

A questionnaire has been designed to measure user salient perceptions of a specific UM device (see Figure 1). The image was used to invoke participant's salient perceptions based purely on what the device affords. Beyond the description of the device as 'wearable' and 'deployable in their work environment', no other information was presented to participants. This was intended to retain the salience of the participant's perceptions and reactions. The device shares a number of affordances with a name badge type object, including wearability and mobility, which we anticipate participants will focus on. One of the benefits of using this image is that participants can cognitively place the device in their own work environments. The device is also presented and introduced as a workplace monitoring system in the same way for all participants.

While there are a number of perceptions in the PSA-BI model [1], it is difficult to explore them all in a single study. As such, a series of 6 prominent perceptions and attitudes were selected for testing (see Table 1 for the corresponding):

- Perceived Natural Border Crossings (PNC): defined as the degree to which a person feels that any natural borders have been crossed [1] , based on Marx [20]. In the context of a monitoring system's, PNC is related to its physical placement in relation to a person.

- Perceived Privacy Invasion (PPI): defined as the degree to which a person feels that the monitoring is invasive of their privacy [1].

- Attitude Toward Technology (ATT): defined as a person's positive or negative view of a technology based on what it affords [1].

- Attitude Toward Behavior (ATB): defined as a person's positive or negative view toward performing a specific behavior related to the technology e.g. use [1].

- $\quad$ Facilitating Conditions (FC): defined as the degree to which an individual believes that the conditions exist which gives them control (or choice) over whether they perform a behavior [8]. For example, if the behavior were use of a wearable device, then the facilitating conditions would be related to whether or not a person is able to remove it.

- Behavioral Intention (BI): defined as an individual's readiness to perform a given behavior, and acts as the direct antecedent of behavior according to the TPB [21]. The behavioral intention under investigation in this study is whether or not a participant would wear the tag presented in Figure 1.

The hypothesized (and tested) relationships between each of these components, as determined by the PSA-BI model [1], can be seen in Figure 2. These hypotheses are grounded in evidence from the ubiquitous computing and surveillance/monitoring literature. One of the main hypotheses for this study is that the Japanese participants are expected to have positive attitudes and intentions toward the wearable workplace UM systems. This is based on existing work practices (i.e. observation in open plan offices) and a protechnology attitude. 


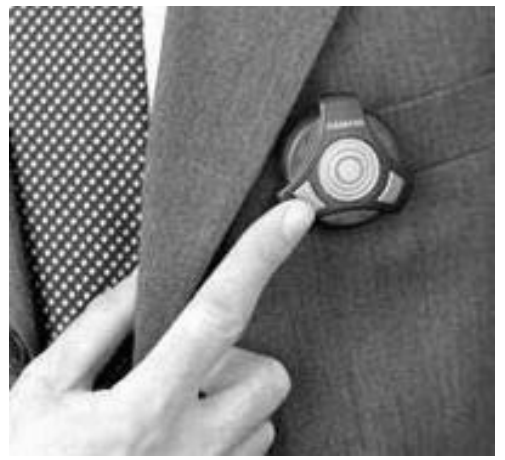

Figure 1. Wearable Ubiquitous Monitoring Device

\section{A. Measure Assessment and Sample Distribution}

A series of three 7 point Likert items were adapted from previous research [1] for each factor (see Table 1). The letter ' $r$ ' indicates that this measure was reversed during statistical analysis, and was included as a negative/positive wording to prevent acquiescence bias. The measures were professionally translated into Japanese, and then crosschecked by both native and foreign fluent Japanese speakers. The items are designed to measure perceptions and attitudes related to the device in Figure 1.

A paper based questionnaire was distributed to 500 office based administrative and support staff at Kyoto University,
Japan. A total of 164 useable responses were received, giving a $33 \%$ response rate. With recommendations of 15 respondents per parameter, this sample size is considered sufficient for a structural equation modeling (SEM) analysis [22]. There was an equal 50\% split between male and female responders. The majority of respondents $(92.7 \%)$ work in open plan offices, most participants $(81.0 \%)$ work full time, and the most common (92.1\%) reported computer skill level was between Novice and Intermediate (a modest response). The measures were assessed for both convergent and discriminant validity. Convergent validity was demonstrated by all factor loadings and average variance extracted (AVE) values being $>0.5$, and all composite reliability and alphas are $\geq 0.7$ [22] (see Table 2). Alpha measures for BI were reduced by the inclusion of Bil, so it was removed to improve the reliability.

Discriminant validity was confirmed by values of AVE > 0.5 , and the square roots of AVE being higher than all other correlations among constructs [22] (see Table 3). However, it should be noted that measure for ATB showed weak discriminant validity, but was still included in the analysis.

SEM simplifies the exploration of multiple hypotheses [22] making it an ideal approach to test the relationships selected from the PSA-BI model. Given that the measures were not normally distributed, the Generalized Least Squares (GLS) statistic was used to estimate the structural model as it is unaffected by non-normality [22].

TABLE I. JAPANESE MEASURES WITH ENGLISH TRANSLATION

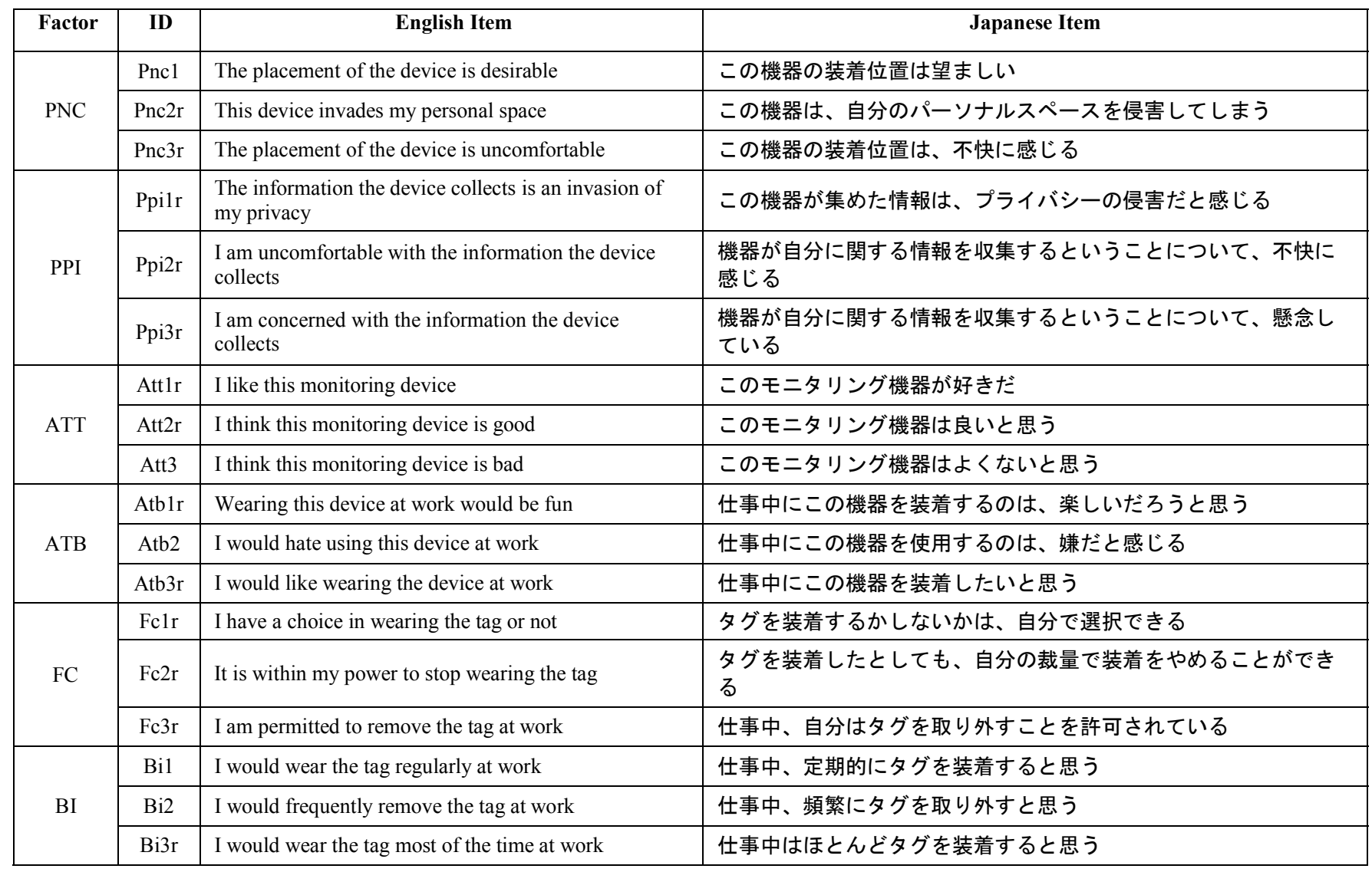




\section{RESULTS AND DISCUSSION}

TABLE II. ASSESSMENT OF CONVERGENT VALIDITY

\begin{tabular}{|c|c|c|c|c|c|c|}
\hline Factor & Item & $\begin{array}{c}\text { Item } \\
\text { Loading }\end{array}$ & Mean & AVE & CR & Alpha \\
\hline \multirow{3}{*}{ PNC } & Pnc1 & .748 & 3.28 & \multirow{3}{*}{.570} & \multirow{3}{*}{.690} & \multirow{3}{*}{.817} \\
\hline & Pnc2r & .772 & 3.78 & & & \\
\hline & Pnc3r & .743 & 3.56 & & & \\
\hline \multirow{3}{*}{ PPI } & Ppilr & .784 & 5.35 & \multirow{3}{*}{.740} & \multirow{3}{*}{.860} & \multirow{3}{*}{.907} \\
\hline & Ppi2r & .901 & 5.47 & & & \\
\hline & Ppi3r & .889 & 5.43 & & & \\
\hline \multirow{3}{*}{$\mathrm{ATT}$} & Att1 r & .853 & 2.51 & \multirow{3}{*}{.650} & \multirow{3}{*}{.780} & \multirow{3}{*}{.832} \\
\hline & Att2r & .850 & 2.96 & & & \\
\hline & Att3 & .705 & 3.34 & & & \\
\hline \multirow{3}{*}{ ATB } & Atb1 $\mathbf{r}$ & .730 & 1.96 & \multirow{3}{*}{.540} & \multirow{3}{*}{.650} & \multirow{3}{*}{.757} \\
\hline & Atb2 & .819 & 2.90 & & & \\
\hline & Atb3r & .640 & 2.10 & & & \\
\hline \multirow{3}{*}{$\mathrm{FC}$} & Fc1r & .846 & 4.78 & \multirow{3}{*}{.800} & \multirow{3}{*}{.910} & \multirow{3}{*}{.926} \\
\hline & $\mathrm{Fc} 2 \mathbf{r}$ & .967 & 4.82 & & & \\
\hline & $\mathrm{Fc} 3 \mathbf{r}$ & .865 & 4.51 & & & \\
\hline \multirow{3}{*}{ BI } & Bil & Excluded & Excluded & \multirow{3}{*}{.540} & \multirow{3}{*}{.550} & \multirow{3}{*}{.697} \\
\hline & $\mathrm{Bi} 2$ & 0.792 & 3.87 & & & \\
\hline & $\mathrm{Bi} 3 \mathrm{r}$ & 0.66 & 4.513 .69 & & & \\
\hline
\end{tabular}

TABLE III. ASSESSMENT OF DISCRIMINANT VALIDITY $($ DIAG. $=\sqrt{ }$ AVE)

\begin{tabular}{|r|c|c|c|c|c|c|}
\hline$p<0.01$ & PNC & PPI & ATT & ATB & FC & BI \\
\hline PNC & .750 & & & & & \\
\hline PPI & .359 & .860 & & & & \\
\hline ATT & -.346 & -.441 & .810 & & & \\
\hline ATB & -.285 & -.554 & .671 & .540 & & \\
\hline FC & .048 & -.048 & .132 & .158 & .890 & \\
\hline BI & -.426 & -.389 & .405 & .402 & -.224 & .730 \\
\hline
\end{tabular}

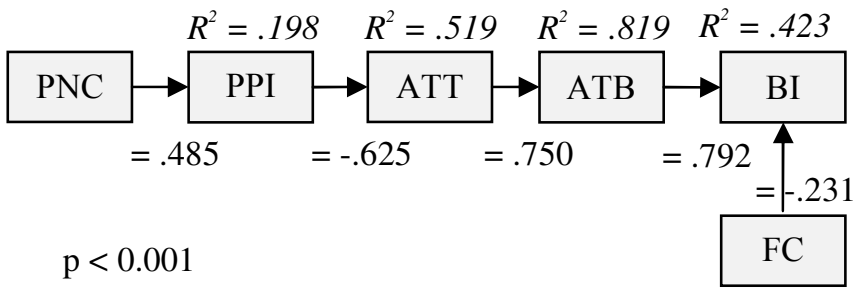

Figure 2. Structural model including parameter estimates and variance
The structural model produced by AMOS is shown in Figure 2. All of the hypothesized relationships were met, with strong estimates for relations at the $\mathrm{p}<0.001$ level. The regression coefficients $(\beta)$ are unstandardized and $\mathrm{R}^{2}$ shows the variance. The GFI (0.864) and RMSEA (0.58) measures were used to assess how well the model fits the data, and indicated a medium to good fitting model [22].

Looking at the structural model, PNC showed a medium strength $(\beta=.485)$ positive relationship with PPI, but only explained $20 \%$ of the variance. It is interesting to observe that the Japanese respondents felt the device would be physically invasive given that Japan is often perceived as more technologically orientated than other cultures. While interesting, it is not necessarily surprising given the findings reaffirm the relationship between physical and privacy invasions found in other research [23].

PPI displayed a strong negative relationship $(=-.625)$ with ATT, explaining 52\% of the variance. This shows that the more invasive of privacy the device was perceived to be, the less positive a respondent's attitude was toward it. Again, as Japan has a wide acceptance of technology, privacy invasion in this instance appears to be influencing the generally positive attitudes toward technology. One reason for this might be the lack of explicit justification.

ATT shows a strong relationship with ATB (=.750), explaining $82 \%$ of the variance. The behavior in this study (wearing the tag) is strongly associated with the technology itself. The salient characteristics of the system (e.g. position on the body) are influencing the attitude towards the technology itself, which are in turn influencing attitudes towards the way the device is intended to be used. This demonstrates the propagation of influence through the model, which is further supported by the strong relationship between ATB and BI ( =.792); as anticipated based on the TPB [8].

There is also a weak relationship $(=-.231)$ between FC and BI, where the more control/choice a person feels they have in wearing the device, the less likely they are to wear it. This is a particularly interesting result, as Japanese people frequently comply with obligations when their behaviour (in this case wearing/not wearing the tag) is visible [24]. This suggests that participants salient perception is that they are not obliged to where the tag. We are likely to see different values for this relationship if the tag were strongly justified or enforced for use in the workplace. Given attitudes towards the device, it is apparent that participants who felt they had a choice in wearing the tag would likely exercise it. Combined, FC and ATB explain $42 \%$ variance in BI, which suggests that the other factors in the PSA-BI model may be important in explaining the remaining variance.

The study is limited in a number of ways: the hypothetical introduction of a device may not have invoked realistic responses from participants, although participants were able to cognitively place the device in their own environments. Secondly, it should be acknowledged that the sample size and response rate were small, limiting the generalizability of the results. However, the sample was sufficient to justifiably perform an analysis using SEM, and provides some insights 
into Japanese perceptions of workplace UM systems. Finally the prediction of the behavioral intention to wear the tag was not validated, which will require further studies.

Aside of these limitations, a number of important conclusions can be drawn from the first part of this study. It is interesting to observe that there are clear patterns in participant's salient perceptions of the device. It suggests that people, when first presented with a device, think along similar lines. A better understanding of salient perceptions could lead to more effective ways of introducing technology to new users. The results also provides an interesting insight into how Japanese people negatively view and, saliently perceive, monitoring technologies in the workplace, where personal space and privacy invasion appear to be prominent issues. This is in direct contrast to what we expected, which was a more positive and open view to the technology.

\section{FUTURE WORK}

The next steps are to make a direct comparison between the salient perceptions of the device by office workers in Kyoto University, with another study exploring the salient perceptions of office workers in the University of Reading, UK. The long-term aim of research involving the PSA-BI model is to fully test all the relationships with a variety of technologies, contexts and participants.

\section{CONCLUSIONS}

Monitoring devices are known to influence the behavior of those being observed, potentially causing issues with the data collected. This is a serious problem for Ubiquitous Computing, and motivates the need for the predictive PSA-BI model. This paper describes the first part of a study exploring the influence of culture as an exogenous moderating variable on perceptions of a wearable monitoring system. The results show signs that those workers in the Kyoto University, Japan, saliently perceive the UM system negatively. A greater understanding of these perceptions could lead to more effective ways of introducing technology to new users, improving the user's subsequent experiences.

\section{REFERENCES}

[1] S. Moran, "User Perceptions of System Attributes in Ubiquitous Monitoring: Toward a Model of Behavioural Intention," Ph.D. Thesis, in School of Construction Management and Engineering, and Informatics Research Centre Reading: University of Reading, 2011, p. 298.

[2] M. Vorvoreanu and C. H. Botan, "Examining Electronic Surveillance In The Workplace: A Review Of Theoretical Perspectives And Research Findings," in the Conference of the International Communication Association Acapulco, Mexico, 2000.

[3] S. Dawson, B. Burnett, and F. McArdle, "Watching Learning From Behind Closed Doors: The Impact of Surveillance on Student Online Behaviour," in ELearn 2005: World conference on E-learning in corporate, government, healthcare and higher education, G. Richards, Ed. Vancouver, Canada: AACE, 2005, pp. 1978-1985.
[4] R. C. Grant, C. A. Higgins, and R. H. Irving, "Computerized performance monitoring systems: Are they costing you customers?," Sloan Management Review, vol. 29, pp. 39-45, 1988.

[5] J. L. Jespersen, A. Albrechtslund, P. Øhrstrøm, P. Hasle, and J. Albretsen, "Surveillance, Persuasion, and Panopticon " Lecture Notes in Computer Science, vol. 4744, pp. 109-120, 2007.

[6] S. Spiekermann, User Control in Ubiquitous Computing: Design Alternatives and User Acceptance: Shaker, Aachen, 2008.

[7] D. Zweig and J. Webster, "Where is the line between benign and invasive? An examination of psychological barriers to the acceptance of awareness monitoring systems," Journal of Organizational Behavior, vol. 23, pp. 605-633, 2002.

[8] I. Ajzen, "The Theory of Planned Behavior," Organizational Behaviour and Human Decision Processes, vol. 50, pp. 179-211, 1991.

[9] F. D. Davis, "Perceived usefulness, perceived ease of use, and user acceptance of information technology," MIS Quarterly, vol. 13, pp. 319340, 1989.

[10] S. Moran, L. Chidzambwa, K. Nakata, and K. Liu, "Assessment of Assistive Technologies based on the PSA-BI Model," in International Conference on PErvasive Technologies Related to Assistive Environments (PETRA '11) Crete, Greece, 2011.

[11] S. Moran, I. Wiafe, and K. Nakata, "User Perceptions of Ubiquitous Monitoring as a Persuasive Strategy," in Fifth International Workshop on Human Aspects In Ambient Intelligence as a part of the International Conference on Intelligent Agent Technology (IAT'11) Campus Scientifique de la Doua, Lyon, France, 2011.

[12] S. J. Barnes and S. L. Huff, "Rising Sun: IMode and the Wireless Internet," Communications of the ACM, vol. 46, pp. 78-84, 2003.

[13] M. Cole, "Signage and Surveillance: Interrogating the Textual Context of CCTV in the UK," Surveillance \& Society: CCTV Special (eds. Norris, McCahill and Wood), vol. 2, pp. 430-445, 2001.

[14] N. Komiya, "A cultural study of the low crime rate in Japan," British Journal of Criminology, vol. 39, pp. 369-390, June 1, 19991999.

[15] R. Ozaki and J. R. Lewis, "Boundaries and the meaning of social space: a study of Japanese house plans," Environment and Planning D: Society and Space, vol. 24, pp. 91-104, 2006.

[16] E. Nagy, S. Yasunaga, and S. Kose, "Japanese Office Employees Psychological Reactions to their Underground and Above-Ground Offices " Journal of Environmental Pyschology, vol. 15, pp. 123-134, 1995.

[17] P. Chee Wei, J. Sutanto, A. Kankanhalli, L. Yan, B. C. Y. Tan, and T. Hock-Hai, "Senior Citizens' Acceptance of Information Systems: A Study in the Context of e-Government Services," Engineering Management, IEEE Transactions on, vol. 53, pp. 555-569, 2006.

[18] M. Y. Yi, K. D. Fiedler, and J. S. Park, "Understanding the Role of Individual Innovativeness in the Acceptance of IT-Based Innovations: Comparative Analyses of Models and Measures," Decision Sciences, vol. 37, pp. 393-426, 2006.

[19] D. H. Nguyen, A. Kobsa, and G. R. Hayes, "An empirical investigation of concerns of everyday tracking and recording technologies," in Proceedings of the 10th international conference on Ubiquitous computing Seoul, Korea: ACM, 2008, pp. 182-191.

[20] G. T. Marx, "Murky Conceptual Waters: the Public and the Private " Ethics and Information Technology, vol. 3, pp. 157-169, 2001.

[21] I. Ajzen and M. Fishbein, Understanding Attitudes and predicting Social Behavior: Prentice-Hall, 1980.

[22] Hair, Anderson, Tatham, and Black, Multivariate Data Analysis, Fifth ed. Upper Saddle River, New Jersey: Prentice-Hall, Inc, 1995.

[23] C. Bryant, "Privacy, privatisation and self-determination," Privacy, Chichester and New York: Wiley, 1978.

[24] M. Hechter and S. Kanazawa, "Group Solidarity and Social Order in Japan," Journal of Theoretical Politcis, vol. 5, pp. 455-493, 1993. 